\title{
Evaluation of Capilia TB assay for rapid identification of Mycobacterium tuberculosis complex in BACTEC MGIT 960 and BACTEC 9120 blood cultures
}

Christopher Muchwa', Joseph Akol ${ }^{1}$, Alfred Etwom', Karen Morgan ${ }^{1}$, Patrick Orikiriza', Francis Mumbowa ${ }^{1,2}$, Paul R Odong ${ }^{1,2}$, David P Kateete ${ }^{2}$, Kathleen D Eisenach ${ }^{3}$ and Moses L Joloba2*

\begin{abstract}
Background: Capilia TB is a simple immunochromatographic assay based on the detection of MPB64 antigen specifically secreted by the Mycobacterium tuberculosis complex (MTC). Capilia TB was evaluated for rapid identification of MTC from BACTEC MGIT 960 and BACTEC 9120 systems in Kampala, Uganda. Since most studies have mainly dealt with respiratory samples, the performance of Capilia TB on blood culture samples was also evaluated.

Methods: One thousand samples from pulmonary and disseminated tuberculosis (TB) suspects admitted to the JCRC clinic and the TB wards at Old Mulago hospital in Kampala, Uganda, were cultured in automated BACTEC MGIT 960 and BACTEC 9120 blood culture systems. BACTEC-positive samples were screened for purity by sub-culturing on blood agar plates. Two hundred and fifty three (253) samples with Acid fast bacilli (AFB, 174 BACTEC MGIT 960 and 79 BACTEC 9120 blood cultures) were analyzed for presence of MTC using Capilia TB and in-house PCR assays.
\end{abstract}

Results: The overall Sensitivity, Specificity, Positive and Negative Predictive values, and Kappa statistic for Capilia TB assay for identification of MTC were $98.4 \%, 97.6 \%, 97.7 \%, 98.4 \%$ and 0.96 , respectively. Initially, the performance of in-house PCR on BACTEC 9120 blood cultures was poor (Sensitivity, Specificity, PPV, NPV and Kappa statistic of 100\%, 29.3\%,7\%, 100\% and 0.04, respectively) but improved upon sub-culturing on solid medium (Middlebrook $7 \mathrm{H} 10$ ) to $100 \%, 95.6 \%, 98.2 \%, 100 \%$ and 0.98 , respectively. In contrast, the Sensitivity and Specificity of Capilia TB assay was $98.4 \%$ and $97.9 \%$, respectively, both with BACTEC blood cultures and Middlebrook $7 \mathrm{H} 10$ cultured samples, revealing that Capilia was better than in-house PCR for identification of MTC in blood cultures. Additionally, Capilia TB was cheaper than in-house PCR for individual samples (\$2.03 vs. \$12.59, respectively), and was easier to perform with a shorter turnaround time (20 min vs. 480 min, respectively).

Conclusion: Capilia TB assay is faster and cheaper than in-house PCR for rapid identification of MTC from BACTEC MGIT 960 and BACTEC 9120 culture systems in real-time testing of AFB positive cultures.

\section{Background}

Genetically related species of the Mycobacterium tuberculosis complex (MTC; M. tuberculosis, M. bovis, M. bovis BCG, $M$. africanum, M. caprae and $M$. cannetti) cause tuberculosis (TB) [1], a global disease that affects one third of the human population $[2,3]$. Tuberculosis

\footnotetext{
*Correspondence: moses.joloba@case.edu

${ }^{2}$ Department of Medical Microbiology, School of Biomedical Sciences,

Makerere University College of Health Sciences, Kampala, Uganda

Full list of author information is available at the end of the article
}

and HIV form a deadly synergy [4] with approx. $75 \%$ of people with HIV/TB co-infection living in sub-Saharan Africa $[2,5]$. Of the 22 high TB burdened countries, Uganda now ranks 16 with an estimated incidence of 452 cases per 100,000 individuals [3]. Kampala, the capital of Uganda with approx. 2 million people, accounts for $\sim 30 \%$ of the nation's TB burden [6].

Accurate diagnosis of TB is crucial for efficient patient management; however, conventional approaches to TB diagnosis still rely on tests with major limitations [7-10].

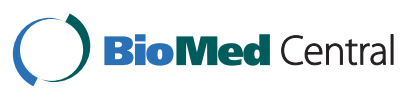


Smear microscopy, a widely available diagnostic method, has low sensitivity ( $30 \%$ to $60 \%$ ) especially in patients coinfected with HIV. The chest X-ray, often used as a supplementary test in smear-negative pulmonary TB also has low specificity. Solid culture as a confirmatory test is expensive, lengthy (up to 8 weeks) and is not widely available in resource limited settings [11]. The World Health Organization (WHO) recommends use of liquid cultures in high TB burdened countries due to advantages of rapid detection and incremental yield in comparison with the solid media [12]. However, liquid culture methods are prone to contamination and usually support growth of nontuberculous mycobacteria (NTM), which may as well inhabit the upper respiratory tract and cause disease in immunocompromised patients [13]. This may lead to reporting false results especially during drug sensitivity testing in that NTM are inherently resistant to common anti-TB drugs $[14,15]$. Further, MTC and NTM cause clinically different clinical symptoms hence prompt identification is crucial for appropriate patient management [16-18].

Recently, nucleic acid amplification tests (NAAT) have been introduced for rapid identification of mycobacteria directly in sample or culture. As such, an in-house PCR assay for identification of MTC was introduced at the Joint Clinical Research Centre (JCRC) in Kampala, Uganda. However, there have been some shortcomings with this method: turnaround time is long ( $\sim 8$ hours) leading to delays in reporting results. Further, we have encountered high rates of false negatives with blood cultures (i.e., templates prepared from BACTEC 9120 system, unpublished observations). Conventional molecular methods are still technologically expensive: reagents require cold storage and shipping; methods are laborintensive and require separate rooms for DNA extraction, amplification and detection. Batching of samples is usually required for cost effectiveness.

Capilia TB assay is an immunochromatographic method that detects MPB64 protein secreted from MTC bacilli into the culture medium [12]. Originally found in M. bovis, similar proteins (i.e. orthologous to MPB64) have been detected in all MTC species and are reportedly rare in NTM. Capilia TB assay is rapid, simple and does not require special equipment $[19,20]$; it has been found efficient for identification of MTC in South Africa, Thailand and Zambia [20,21]. In this study, the performance of Capilia TB assay was evaluated for rapid detection of MTC from BACTEC MGIT 960 and BACTEC 9120 systems in Kampala, Uganda. Additionally, the performance of Capilia TB on blood cultures was evaluated since previous studies mainly dealt with respiratory samples. The overall Sensitivity, Specificity, PPV and NPV of Capilia TB assay were high and in agreement with values obtained elsewhere.

\section{Methods}

\section{Study setting and design}

Samples for this cross sectional study were obtained from pulmonary and disseminated TB suspects (at baseline, follow up and retreatment cases) admitted to the JCRC clinic and the TB wards at Old Mulago hospital in Kampala, Uganda, from April 2008 to May 2009. Sputum and gastric samples in sterile $50 \mathrm{ml}$ Falcon tubes were obtained directly from TB suspects and injected into sterile $50 \mathrm{ml}$ Falcon tubes while blood samples were drawn intravenously by a study nurse and inoculated into BACTEC 9120 blood culture bottles. Samples were cultured in automated BACTEC MGIT 960 and BACTEC 9120 systems (samples from Old Mulago hospital were transported daily by courier to the JCRC for culture). A convenient sample size (i.e. which did not affect patient management) of 253 AFB-positive blinded samples was considered; these were selected in batches of 30 upon detection of AFB (within 42 days; AFB-negative samples in the same period were excluded). In-house PCRs were performed at the Molecular Biology Laboratory, Makerere University College of Health Sciences (MakCHS) while Capilia TB assays were performed at the JCRC.

\section{Sample processing and culture}

Sputum and gastric samples were processed in Biosafety cabinet class II following the standard N-Acetyl Lcysteine (NALC)/NaOH method [22]. Briefly, an equal volume of decontamination/digestion buffer $(6 \% \mathrm{NaOH}$, $2.9 \%$ Na-citrate and NALC) was added to each sample, vortexed for $5 \mathrm{~min}$ and incubated at room temperature for $15 \mathrm{~min}$. The digested samples were neutralized with phosphate buffer ( $\mathrm{pH}$ 6.8) and mixed thoroughly by inversion. The samples were centrifuged at $3000 \mathrm{~g}$ for 15 minutes, and the sediment suspended in $2.5 \mathrm{ml}$ phosphate buffer ( $\mathrm{pH} 6.8$ ). Then, $0.8 \mathrm{ml}$ of the growth supplement, Polymxin B, Amphoterin B, Nalidixic acid, Trimetroprim and Azlocillin (PANTA) was added to the Mycobacterium Growth Indicator Tubes (MGIT), to which $0.5 \mathrm{ml}$ of the digested sample was added and incubated in BACTEC MGIT 960 system for 6 weeks. For blood cultures, the barcodes were scanned prior to incubating directly for 6 weeks in the BACTEC 9120 system. Ziehl Neelsen (ZN) smears were performed on BACTEC MGIT 960 and BACTEC 9120 samples with microbial growth to determine the presence of AFB. Purity checks were performed by sub-culturing on blood agar plates.

\section{Capilia TB assays}

Capilia TB assay (TAUN, Numazu, Japan) was performed on AFB-positive BACTEC samples following the 
manufacturer's guidelines. For bacterial colonies that grew on solid media (Middlebrook 7H10), a suspension was made by mixing a single colony with $0.2 \mathrm{ml}$ extraction buffer and vortexed; the resultant suspension was then applied onto the Capilia TB strip (following the manufacture's guidelines).

\section{In-house PCR}

The performance of Capilia TB assay was compared with that of in-house PCR, which we used as a baseline identification test for MTC. The in-house PCR protocol was based on the detection of IS6110, which is unique to members of the MTC [22]. Templates were prepared from BACTEC cultures and used in a PCR-amplification procedure previously described [22]. Furthermore, MTC were identified to species level using the regions of difference (RD) genotyping method as previously described by Asiimwe et al, 2008 [23]. All the strains belonged to M. tuberculosis sensu strict [23].

\section{Quality control}

To avoid cross contamination, separate rooms and equipment were used for sample preparation, DNA extraction and preparation of amplification reactions. In addition, positive and negative controls were always included in the PCRs. Amplification reactions and PCR products were opened in separate UV hoods each located in separate rooms. For cultures, aerosol resistant tips 160 were used and changed for each sample as previously described [22]. Samples with discrepant 161 results were analyzed with the Hains MTB identification kit (Hains life sciences, Nehren, 162 Germany). Blood samples in which AFB grew were further sub-cultured on Middlebrook 7H10 163 agar and analyzed again with both in-house PCR and Capilia TB assay.

\section{Ethical consideration}

Ethical approval was obtained from the institutional review board of the JCRC and the Uganda National Council for Science and Technology. Written informed consent was obtained from all the study participants.

\section{Results and discussion}

\section{Performance of capilia TB for identification of MTC in BACTEC cultures}

In this study, the overall Sensitivity, Specificity, PPV and NPV of Capilia TB assay were high and in agreement with values obtained in other studies $[17,20,21,24]$. Of the 253 AFB positive samples, $79(31 \%, 79 / 253)$ were BACTEC 9120 blood cultures while 174 (69\%, 174/253) were BACTEC MGIT 960 cultures. M. tuberculosis complex bacilli were identified in 129 samples (51\%, $129 / 253)$ by Capilia TB and in 130 (51\%, 130/253) by in-house PCR. Capilia identified 124 (49\%, 124/253)
NTM while 130 (51\%, 130/253) were identified by inhouse PCR. The overall sensitivity, specificity, PPV and NPV of Capilia TB assay were 98.4\%, 97.6\%, 97.7\%, and $18098.4 \%$, respectively (Table 1). The Kappa statistic was 0.96 indicating almost perfect agreement between the tests.

Performance of capilia TB assay for identification of MTC in pure vs. Contaminated cultures

Of the 253 BACTEC AFB-positive cultures, 139 (55\%, $139 / 253)$ were contaminated while 114 (45\%, 114/253) were pure. Screening contaminated cultures (see Table 2) with Capilia TB assay for MTC revealed Sensitivity, Specificity, PPV and NPV of 95.1\%, 99\%, 97.5\% and 98\% respectively, which values were in a similar range with those for pure cultures (i.e., 98.9\%, 96.3\%, 98.9\% and 96.3\% respectively). The Kappa statistics for both tests in contaminated and pure samples were 0.98 and 0.95 (almost perfect agreement), respectively.

\section{Performance of capilia TB in identification of MTC from}

\section{BACTEC blood cultures and sub-cultures on solid media}

Prior to sub-culture on Middle brook $7 \mathrm{H} 10$ plates, 57 $(72 \%, 57 / 79)$ of the 79 AFB-positive BACTEC 9120 blood cultures were identified as MTC by Capilia TB assay while only four $(5 \%, 4 / 79)$ were identified by inhouse PCR. Additionally, Capilia TB identified 22 (28\%, 22/79) blood cultures as NTM while in-house PCR identified 75 (95\%, 75/79). The Sensitivity, Specificity, PPV and NPV of Capilia TB assay on direct blood cultures were $100 \%, 29.3 \%, 7 \%$ and $100 \%$ respectively (Table 3). The Kappa statistic was 0.04, indicating slight agreement between capilia and in-house PCR when used on direct blood culture. Following sub-culturing on Middlebrook $7 \mathrm{H} 10$ plates, Capilia TB identified the same number of samples as MTC (i.e. 57/79, 72\%) while in-house PCR identified 56 (i.e. 52 more, 71\%). When the initial in-house PCR negative blood cultures were tested by the Hains MTB test, 57 (72\%, 57/79) samples were confirmed as MTC, which was $100 \%$ in agreement with the Capilia TB assay. For in-house PCR, the specificity (29.3\% vs. $95.6 \%)$ and PPV (7\% vs. $98.2 \%)$ improved

Table 1 Sensitivity and Specificity of Capilia TB in comparison with in-house PCR

\begin{tabular}{lllll}
\hline \multirow{2}{*}{ Capilia } & \multicolumn{5}{c}{ PCR } \\
\cline { 2 - 5 } & \multicolumn{1}{c}{ Positive } & Negative & Total \\
\cline { 2 - 5 } & Positive & 127 & 2 & 129 \\
\cline { 2 - 5 } & Negative & 3 & 121 & 124 \\
\cline { 2 - 5 } & Total & 130 & 123 & 253 \\
\hline
\end{tabular}

Sensitivity $(\mathrm{Se})=98.4 \%$; Specificity $(\mathrm{Sp})=97.58 \%$; Positive predictive value $(\mathrm{PPV})=97.7 \%$; Negative predictive value (NPV) $=98.4 \%$ and Kappa statistic (\#) $=0.96$ (almost perfect) 
Table 2 Performance of Capilia TB for identification of MTC on contaminated and pure BACTEC cultures

\begin{tabular}{|c|c|c|c|c|}
\hline \multirow[b]{2}{*}{${ }^{\mathrm{a} C}$ Capilia } & \multicolumn{4}{|c|}{ PCR } \\
\hline & & Positive & Negative & Total \\
\hline & Positive & 39 & 1 & 40 \\
\hline & Negative & 2 & 97 & 99 \\
\hline & Total & 41 & 98 & 139 \\
\hline \multirow[t]{3}{*}{${ }^{b}$ Capilia } & Positive & 86 & 1 & 87 \\
\hline & Negative & 1 & 26 & 27 \\
\hline & Total & 87 & 27 & 114 \\
\hline
\end{tabular}

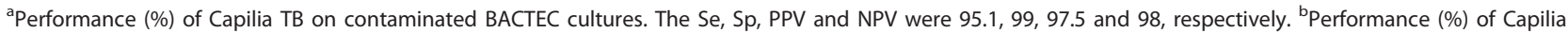
TB in pure BACTEC cultures. The Se, Sp, PPV, NPV \& (\#) were 98.9, 96.3, 98.9, 96.3, and (0.98 \& 0.95), repectively, (almost perfect agreement)

Table 3 Performance of Capilia TB for identification of MTC in direct blood cultures and on solid media (7H10 subcultures)

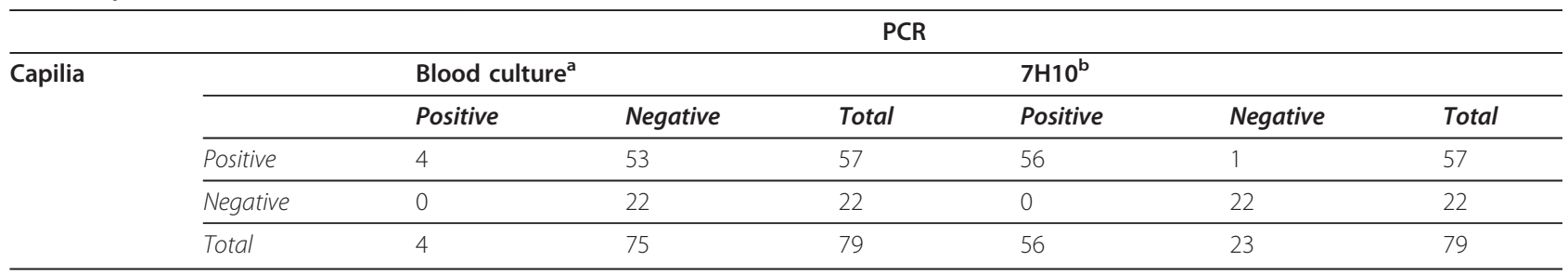

${ }^{\mathrm{a}}$ The performance (\%) of Capilia TB on templates picked directly from blood cultures. The Se, Sp, PPV and NPV were, $100,29,7$ and 100, respectively.

berformance (\%) on Capilia TB assay on Middlebrook 7H10 media. The Se, Sp, PPV, NPV \& (\#) were 100, 95.6, 98.2, 100 and (0.98), respectively (almost perfect agreement)

only after sub-culturing on Middlebrook 7H10 plates, whereas values for Capilia TB remained unchanged (Table 3). This confirmed presence of MTC in the 57 blood culture samples as initially identified by Capilia TB assay. The agreement between the two tests was almost perfect (kappa statistic $=0.98)$. Thus, Capilia TB assay was more efficient than in-house PCR at identifying MTC in BACTEC blood cultures. This demonstrated the superiority of Capilia TB assay to in-house PCR for identification of MTC in various samples. For in-house PCR, the initial high number of false negatives was probably due to PCR inhibitors in blood (such as haeme and/or porphyrins from lysed erythrocytes believed to inhibit Taq polymerase [25]), since PCRs on
7H10 samples turned positive. In terms of materials and labor, the overall cost per sample for identification of MTC with Capilia TB was cheaper than that for inhouse PCR (Table 4). Moreover, the cost estimations are more expensive even after excluding equipment (such as thermocyclers).

\section{Limitations}

Due to limited resources, we could not sequence-confirm MTC species nor perform biochemical assays, which are regarded as gold standards for MTC identification. Furthermore, AFB were detected with $\mathrm{ZN}$ staining which is not very sensitive, implying that MTC in ZN negative samples were probably missed. Further,

Table 4 Cost per test for performing Capilia TB in comparison with in-house PCR

\begin{tabular}{|c|c|c|c|c|}
\hline \multirow[t]{2}{*}{ Materials per test } & \multicolumn{2}{|c|}{ PCR } & \multicolumn{2}{|l|}{ Capilia } \\
\hline & Quantity & $\operatorname{Cost}^{a}$ & Quantity & Cost $^{a}$ \\
\hline Cryovial & 1 & 0.34 & - & \\
\hline Pasteur pipettes & 1 & 0.19 & 1 & 0.19 \\
\hline Capilia test cassette & - & - & 1 & 1.84 \\
\hline$P C R$ reagents & 1 & 10.00 & - & \\
\hline Quality control PCR & 1 & 0.53 & - & \\
\hline Pipette tips & 8 & 0.40 & - & \\
\hline$P C R$ tubes & 1 & 0.05 & - & \\
\hline Total cost ${ }^{a}$ & & 12.59 & & 2.03 \\
\hline
\end{tabular}

-Not applicable; ${ }^{\text {aUS dollars }}$ 
mutations in the mpb64 gene usually lead to detection of false negatives $[12,24]$ while NTM testing positive with Capilia TB tests have been recently reported $[12,24]$; these, coupled with the failure to distinguish members of the MTC and failure to work on clinical specimens somehow negate the efficiency of Capilia TB assays. These shortcomings are also re-iterated in the manufacturer's kit inserts implying that the kit should be cautiously used.

\section{Conclusion}

Capilia TB assay performed better and was cheaper than IS6110 in-house PCR for rapid identification of MTC from BACTEC MGIT 960 and BACTEC 9120 systems. The optimal performance of in-house PCR on blood cultures requires an additional isolation step on solid media for optimum performance.

\begin{abstract}
Abbreviations
AFB: Acid fast bacilli; MTC: Mycobacterium tuberculosis complex; MTB: Mycobacterium tuberculosis; IS6110: Insertion Sequence 6110; JCRC: Joint Clinical Research Center; NAAT: Nucleic acid amplification tests; MGIT: Mycobacteria growth indicator tube; NTM: Non-tuberculous mycobacteria; PCR: Polymerase chain reaction; TBRU: Tuberculosis Research Unit; ZN: ZiehlNeelsen; PANTA: Polymxin B, amphoterin B, nalidixic acid, trimetroprim and azlocillin; NPV: Negative predictive value; PPV: Positive predictive value; MakCHS: Makerere University College of Health Sciences.
\end{abstract}

\section{Acknowledgements}

The authors thank the Foundation for Innovative New Diagnostics (FIND), Kampala, Uganda and the ZAMBART project, Zambia, for providing Capilia TB kits; and the staff at the JCRC and Molecular labs (MakCHS) for their invaluable support. This project was funded in part by the Tuberculosis Research Unit (TBRU), established with Federal funds from the United Sates National Institutes of Allergy and Infectious Diseases \& the United States National Institutes of Health and Human Services, under Contract Nos. NO1Al-95383 and HHSN266200700022C/NO1-Al-70022.

\section{Author details}

${ }_{1}^{1}$ JCRC TB Laboratory, Joint Clinical Research Center, Mengo, Kampala, Uganda. ${ }^{2}$ Department of Medical Microbiology, School of Biomedical Sciences, Makerere University College of Health Sciences, Kampala, Uganda. ${ }^{3}$ Department of Pathology, University of Arkansas for Medical Sciences, Little Rock, USA

\section{Authors' contributions}

CM carried out the experiments and drafted the manuscript, which was proofread and revised by DPK and AE. JA, FM and KM participated in study design; AE performed the statistical analysis. PRO performed the in-house PCR. CM, KE and MLJ conceived the study, which was supervised by KE and MLJ. All authors read and approved the final manuscript.

\section{Competing interests}

The authors declare that they have no competing interests.

Received: 30 June 2011 Accepted: 19 January 2012

Published: 19 January 2012

\section{References}

1. Cole ST: Comparative and functional genomics of the Mycobacterium tuberculosis complex. Microbiology 2002, 148(Pt 10):2919-2928.

2. Tsiouris SJ, Gandhi NR, El-Sadr WM, Gerald F: Tuberculosis and HIV-Needed A New Paradigm for the Control and Management of Linked Epidemics. J Int AIDS Soc 2007, 9(3):62.
3. World Health Organisation: Global tuberculosis control. A short update to the 2009 report.[http://www.who.int/tb/publications/global_report/2009/ update/en/index.html].

4. The deadly synergy of HIV and tuberculosis. Lancet Infect Dis 2010, 10(7):441.

5. El-Sadr WM, Tsiouris SJ: HIV-associated tuberculosis: diagnostic and treatment challenges. Semin Respir Crit Care Med 2008, 29(5):525-531.

6. Tuberculosis Profile for Uganda - USAID. [http://pdf.usaid.gov/pdf_docs/ PDACl554.pdf].

7. Gagneux S, Burgos MV, DeRiemer K, Enciso A, MuÃoz S, Hopewell PC, Small PM, Pym AS: Impact of Bacterial Genetics on the Transmission of Isoniazid-Resistant Mycobacterium tuberculosis. PLoS Pathog 2006, 2(6): e61.

8. Nahid P, Pai M, Hopewell PC: Advances in the diagnosis and treatment of tuberculosis. Proc Am Thorac Soc 2006, 3(1):103-110.

9. Foulds J, O'Brien R: New tools for the diagnosis of tuberculosis: the perspective of developing countries. Int J Tuberc Lung Dis 1998, 3(10):778-783.

10. Perkins MD: New diagnostic tools for tuberculosis. Int J Tuberc Lung Dis 2000, 4(12 Suppl 2):S182-S188.

11. Reid MJ, Shah NS: Approaches to tuberculosis screening and diagnosis in people with HIV in resource-limited settings. Lancet Infect Dis 2009, 9(3):173-184.

12. Shen GH, Chiou CS, Hu ST, Wu KM, Chen JH: Rapid identification of the Mycobacterium tuberculosis complex by combining the ESAT-6/CFP-10 immunochromatographic assay and smear morphology. J Clin Microbiol 49(3):902-907.

13. Wagner D, Young LS: Nontuberculous mycobacterial infections: a clinical review. Infection 2004, 32(5):257-270.

14. Griffith DE: Nontuberculous mycobacterial lung disease. Curr Opin Infect Dis 2010, 23(2):185-190.

15. Griffith DE, Brown-Elliott BA, Wallace RJ Jr: Diagnosing nontuberculous mycobacterial lung disease. A process in evolution. Infect Dis Clin North Am 2002, 16(1):235-249.

16. Diagnosis and treatment of disease caused by nontuberculous mycobacteria. This official statement of the American Thoracic Society was approved by the Board of Directors, March 1997. Medical Section of the American Lung Association. Am J Respir Crit Care Med 1997, 156(2 Pt 2):S1-25.

17. Hasegawa N, Miura T, Ishii K, Yamaguchi K, Lindner TH, Merritt S, Matthews JD, Siddiqi SH: New simple and rapid test for culture confirmation of Mycobacterium tuberculosis complex: a multicenter study. J Clin Microbiol 2002, 40(3):908-912.

18. Park MY, Kim YJ, Hwang SH, Kim HH, Lee EY, Jeong SH, Chang CL: Evaluation of an immunochromatographic assay kit for rapid identification of Mycobacterium tuberculosis complex in clinical isolates. J Clin Microbiol 2009, 47(2):481-484.

19. Shen $\mathrm{GH}$, Chen $\mathrm{CH}$, Hung $\mathrm{CH}$, Wu KM, Lin CF, Sun YW, Chen JH: Combining the Capilia TB assay with smear morphology for the identification of Mycobacterium tuberculosis complex. Int I Tuberc Lung Dis 2009, 13(3):371-376.

20. Ngamlert K, Sinthuwattanawibool C, McCarthy KD, Sohn H, Starks A, Kanjanamongkolsiri P, Anek-vorapong R, Tasaneeyapan T, Monkongdee P, Diem $L$, et al: Diagnostic performance and costs of Capilia TB for Mycobacterium tuberculosis complex identification from broth-based culture in Bangkok, Thailand. Trop Med Int Health 2009, 14(7):748-753.

21. Muyoyeta M, de Haas PE, Mueller DH, van Helden PD, Mwenge L, Schaap A, Kruger C, van Pittius NC, Lawrence K, Beyers N, et al: Evaluation of the Capilia TB assay for culture confirmation of Mycobacterium tuberculosis infections in Zambia and South Africa. J Clin Microbiol 2010, 48(10):3773-3775.

22. Muhumuza J, Asiimwe BB, Kayes S, Mugyenyi R, Whalen C, Mugerwa RD, Boom $\mathrm{H}$, Eisenach KD, Joloba ML: Introduction of an in-house PCR for routine identification 322 of $\mathrm{M}$. tuberculosis in a low-income country. Int J Tuberc Lung Dis 2006, 10(11):1262-1267.

23. Asiimwe BB, Koivula T, Kallenius G, Huard RC, Ghebremichael S, Asiimwe J, Joloba ML: Mycobacterium tuberculosis Uganda genotype is the predominant cause of TB in Kampala, Uganda. Int J Tuberc Lung Dis 2008, 12(4):386-391.

24. Hirano K, Aono A, Takahashi M, Abe C: Mutations including IS6110 insertion in the gene encoding the MPB64 protein of Capilia TB- 
negative Mycobacterium tuberculosis isolates. J Clin Microbiol 2004, 42(1):390-392

25. Qian Q, Tang YW, Kolbert CP, Torgerson CA, Hughes JG, Vetter EA,

Harmsen WS, Montgomery SO, Cockerill FR, Persing DH: Direct

identification of bacteria from positive blood cultures by amplification and sequencing of the 16S rRNA gene: evaluation of BACTEC 9240 instrument true-positive and false-positive results. J Clin Microbiol 2001, 39(10):3578-3582.

doi:10.1186/1756-0500-5-44

Cite this article as: Muchwa et al:: Evaluation of Capilia TB assay for rapid identification of Mycobacterium tuberculosis complex in BACTEC MGIT 960 and BACTEC 9120 blood cultures. BMC Research Notes 2012 5:44.

Submit your next manuscript to BioMed Central and take full advantage of:

- Convenient online submission

- Thorough peer review

- No space constraints or color figure charges

- Immediate publication on acceptance

- Inclusion in PubMed, CAS, Scopus and Google Scholar

- Research which is freely available for redistribution

Submit your manuscript at www.biomedcentral.com/submit 